\title{
Interaction of Electromagnetic Waves With a Magnetized Nonuniform Plasma Slab
}

\author{
D. L. Tang, A. P. Sun, X. M. Qiu, and Paul K. Chu, Fellow, IEEE
}

\begin{abstract}
The absorption, reflection, and transmission of electromagnetic waves by a nonuniform plasma slab immersed in an ambient uniform magnetic field of various strengths are studied in this paper. The effects of the plasma parameters and magnetic field strength on the absorbed, reflected, and transmitted power are discussed. The magnetized nonuniform plasma slab is modeled by a series of magnetized uniform plasma subslabs. The calculation results show that the effects of the magnetic field strength and density gradient on the absorbed power, as well as the frequency band of resonant absorption, are significant. A complete analysis utilizing the scattering matrix method is also used to compare the above calculation results which neglect multiple reflections between subslab interfaces. Broadband absorption of electromagnetic waves can be achieved by changing the magnetic field strength and plasma density. More than $90 \%$ of the electromagnetic wave power can be absorbed in a magnetized nonuniform plasma slab with width of 12 $\mathrm{cm}$ and the absorption bandwidth can range from 1 to $20 \mathrm{GHz}$ with different plasma parameters and external magnetic field strengths.

PACS: 52.35.Hr, 52.25.Xz, 52.40.Db
\end{abstract}

Index Terms-Electromagnetic waves, plasma slab.

\section{INTRODUCTION}

$\mathbf{T}$ HE INTERACTION of electromagnetic waves with plasma is an interesting problem in plasma physics [1]-[3]. The propagation of electromagnetic waves in low-temperature plasma has attracted much interest in recent years due to the potential applications of atmospheric pressure plasma as reflectors or absorbers of electromagnetic waves [4]-[10]. Vidmar [4] has found that cold, collisional plasma generated at atmospheric pressure can be used as a broadband absorber. Laroussi [8] has numerically investigated the interactions between microwaves and high-atmospheric pressure plasma. Koretzky and Kuo [9] have demonstrated that plasma torches can effectively attenuate microwaves, and Liu et al. [10] have discussed the attenuation property of electromagnetic waves in atmospheric pressure plasma. These research activities have mainly focused on the propagation of electromagnetic waves in unmagnetized plasma. If a magnetic field is applied to the background plasma equilibrium, the characteristics of

Manuscript received October 6, 2002; revised January 23, 2003. This work was supported by the National Natural Science Foundation of China under Grant 10005001 and by the Hong Kong Research Grants Council CERG CityU 1013/01E or 9040577.

D. L. Tang is with the Southwestern Institute of Physics, Chengdu 610041, China. He is also with the Department of Physics and Materials Science, City University of Hong Kong, Kowloon, Hong Kong (e-mail: tangdeli@263.net).

A. P. Sun and X. M. Qiu are with the Southwestern Institute of Physics, Chengdu 610041, China.

P. K. Chu is with the Department of Physics and Materials Science, City University of Hong Kong Kowloon, Hong Kong (e-mail: paul.chu@ @ityu.edu.hk).

Digital Object Identifier 10.1109/TPS.2003.811648

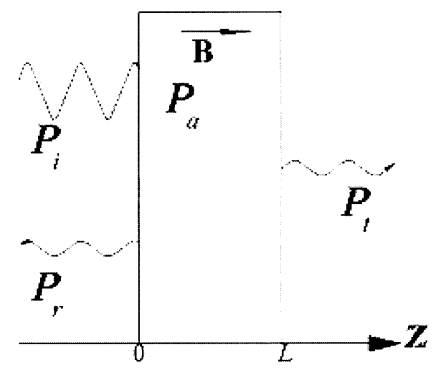

Fig. 1. Schematic diagram of the wave propagation.

the electromagnetic waves propagation in the plasma will be affected by the new dynamical effect of Larmor gyration [11]. Laroussi and Roth [7] have investigated the reflection, absorption, and transmission of microwaves by a magnetized nonuniform plasma slab. Ruck et al. and Kong present the electromagnetic wave reflection from dielectric slab by $2 \times$ 2 matrix approaches [12], [13]. A scattering matrix method (SMM) analysis of the reflection, absorption, and transmission from nonuniform magnetized plasma slab has been reported [14]. Some experimental microwave absorbers have also investigated with regard to the effects of varying magnetic fields [15], [16]. In the work reported in this paper, we investigate the broadband absorption characteristics of magnetized plasma by changing the plasma parameters and uniform magnetic field strengths. SMM analysis is also adopted here for comparison with the calculation results which neglect multiple reflections.

\section{Propagation Properties}

The incident wave is assumed to be a plane wave launched at a normal incident angle to the plasma slab, as shown in Fig. 1. The plane wave propagates parallel to the external magnetic field $\mathbf{B}$. The plasma is assumed to be cold, weakly ionized, steady-state, nonuniform, and collisional. A parabolic plasma density profile is assumed across the slab in the $z$ direction. This parabolic density profile is approximated by an exponential variation $n=n_{c} e^{2(1-2 z / L) / 3}(z>L / 2)$ and $n=n_{c} e^{2(2 z / L-1) / 3}(z<$ $L / 2)$, where $n_{c}$ is the number density at the center of the plasma slab. The interaction of the electromagnetic waves with the magnetized nonuniform plasma is modeled as a series of magnetized uniform plasma subslabs with the wave being absorbed and transmitted in each slab and reflected at the interface of each slab. This model is acceptable as first approximation under the assumption that the plasma properties vary slowly along the wave propagation path [11]. In this model, a plane wave propagating in a plasma subslab obeys the following Maxwell's equa- 
tions and R-wave parallel to the magnetic field is considered in the following analysis:

$$
\begin{aligned}
\nabla \times \mathbf{E} & =-j \omega \mu_{o} \mathbf{H} \\
\nabla \times \mathbf{H} & =\left(\sigma+j \omega \varepsilon_{r} \varepsilon_{o}\right) \mathbf{E}
\end{aligned}
$$

where $\mathbf{J}=\sigma \mathbf{E}$. Here, we assume the time behavior of the wave to be $\exp (j \omega t)$. Equation (2) can be written as

$$
\nabla \times \mathbf{H}=j \omega \widetilde{\varepsilon}_{r} \varepsilon_{o} \mathbf{E}
$$

where $\widetilde{\varepsilon}_{r}$ is the complex dielectric constant. The resulting wave equation is

$$
\nabla^{2} \mathbf{E}=-\frac{\widetilde{\varepsilon}_{r}}{c^{2}} \omega^{2} \mathbf{E}
$$

where $c$ is the speed of light. Here, we use $\nabla \cdot \mathbf{E}=0$ which is true for R-wave. A plane wave propagating in the $z$ direction with a phase dependence of $\exp (j \omega t-\widetilde{\gamma} z)$ is a solution of (4)

$$
\mathbf{E}=\mathbf{E}_{\mathbf{o}} \exp (j \omega t-\tilde{\gamma} z)
$$

where $\widetilde{\gamma}$ is the complex propagation constant. The dispersion characteristic of a plane wave propagating in the magnetized cold plasma subslab is

$$
\widetilde{\gamma}^{2}=-\widetilde{\varepsilon}_{r} \frac{\omega^{2}}{c^{2}}
$$

and $\widetilde{\gamma}$ is generally expressed as

$$
\widetilde{\gamma}=\alpha+j \beta
$$

where $\alpha$ is the attenuation coefficient and $\beta$ is the phase coefficient.

We assume that a plane wave propagates perpendicular to the magnetized plasma slab and parallel to the external magnetic field $\mathbf{B}$. The plasma slab width is $12 \mathrm{~cm}$ and is divided as 12 adjacent two-dimensional plasma subslabs with sharp boundaries. The schematic diagram of the wave propagation characteristics at an arbitrary angle of incidence is depicted in Fig. 2. In each subslab, the plasma number density is assumed to be uniform. At the center of the plasma slab, the plasma density is maximal. The incident wave travels from a subslab to the next one with reflection at the interface. The reflection wave will be partially absorbed by the plasma subslabs before it is reflected back out of the plasma. The total reflected power is calculated by neglecting multiple reflections between subslab interfaces. The total incident power, reflected power, transmitted power, and absorbed power are denoted by $P_{i}, P_{r}, P_{t}$ and $P_{a}$, respectively. The complex dielectric constant is calculated for each sub-slab and the reflection at each interface is obtained. The complex dielectric constant $\widetilde{\varepsilon}_{r}$ for a plane wave propagating through a cold

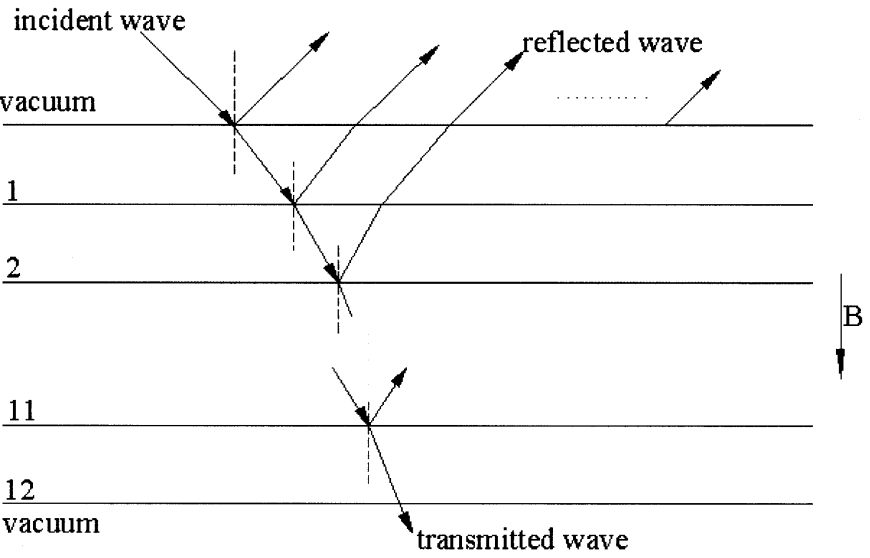

Fig. 2. Schematic diagram of the wave absorption, transmission, and reflection at an arbitrary angle of incidence.

plasma at an arbitrary incident angle has been derived by Appleton [11] (see (8) at the bottom of page) where $\omega_{p}, \omega_{c e}, \nu_{\mathrm{en}}$, $\theta$ are, respectively, the electron plasma frequency, the electron gyrofrequency, the effective collision frequency between electron and neutral gas, and the angle of propagation with respect to the static background magnetic field. The \pm sign indicates left- and right-hand polarization. Here, we assume a right-hand polarization and $\theta=0^{\circ}$. Consequently, (8) becomes

$$
\widetilde{\varepsilon}_{r}=1-\frac{\frac{\omega_{p}^{2}}{\omega^{2}}}{1-j \frac{\nu_{\text {en }}}{\omega}-\frac{\omega_{c e}}{\omega}} .
$$

For a right-hand polarization mode and the normal incident wave, the reflection coefficient on the $i+1$ th interface is

$$
\Gamma(i+1)=\frac{\sqrt{\widetilde{\varepsilon}_{r}(i)}-\sqrt{\widetilde{\varepsilon}_{r}(i+1)}}{\sqrt{\widetilde{\varepsilon}_{r}(i)}+\sqrt{\widetilde{\varepsilon}_{r}(i+1)}} .
$$

By calculating the reflection and absorption power of each subslab, the total reflection power can be obtained as follows:

$$
\begin{aligned}
P_{r}=P_{i} & \left\{|\Gamma(1)|^{2}+\sum_{j=2}^{12}\left(|\Gamma(j)|^{2}\right.\right. \\
& \left.\left.\times \prod_{i=1}^{j-1}\left(\exp [-4 \alpha(i) d]\left(1-|\Gamma(i)|^{2}\right)\right)\right)\right\}
\end{aligned}
$$

where $d$ is the width of a subslab. The total transmitted power is given by

$$
P_{t}=P_{i} \prod_{i=1}^{12}\left(\exp [-2 \alpha(i) d]\left(1-|\Gamma(i)|^{2}\right)\right) .
$$

$$
\widetilde{\varepsilon}_{r}=1-\frac{\frac{\omega_{p}^{2}}{\omega^{2}}}{\left[1-j \frac{\nu_{\mathrm{en}}}{\omega}-\frac{\frac{\omega_{c e}^{2}}{\omega^{2}} \sin ^{2} \theta}{2\left(1-\frac{\omega_{p}^{2}}{\omega^{2}}-j \frac{\nu_{\mathrm{en}}}{\omega}\right)}\right] \pm\left[\frac{\frac{\omega_{c e}^{4}}{\omega^{4}} \sin ^{4} \theta}{4\left(1-\frac{\omega_{p}^{2}}{\omega^{2}}-j \frac{\nu_{\mathrm{en}}}{\omega}\right)^{2}}+\frac{\omega_{c e}^{2}}{\omega^{2}} \cos ^{2} \theta\right]^{\frac{1}{2}}}
$$




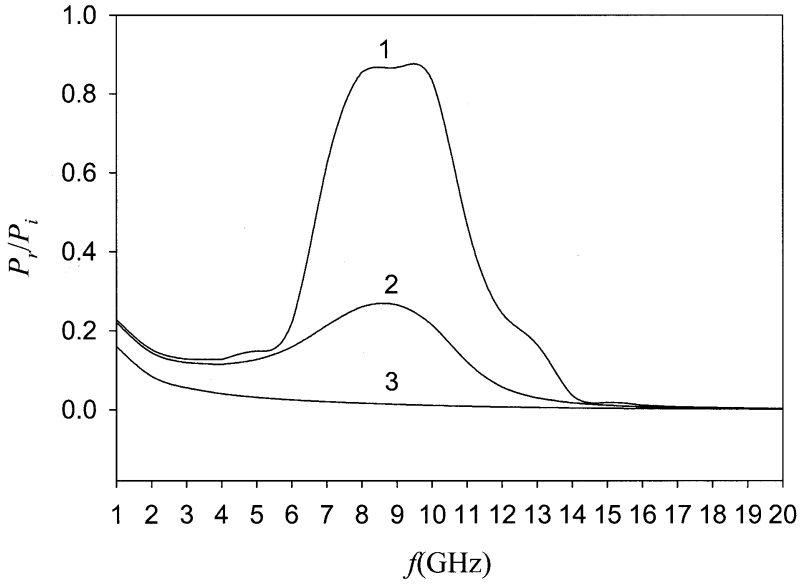

Fig. 3. Reflection ratio versus wave frequency, $n_{c}=1 \times 10^{18} \mathrm{~m}^{-3}, B=$ $2.5 \mathrm{kG}$. (1) $\nu_{\mathrm{en}}=1 \mathrm{GHz}$. (2) $\nu_{\mathrm{en}}=5 \mathrm{GHz}$. (3) $\nu_{\mathrm{en}}=60 \mathrm{GHz}$.

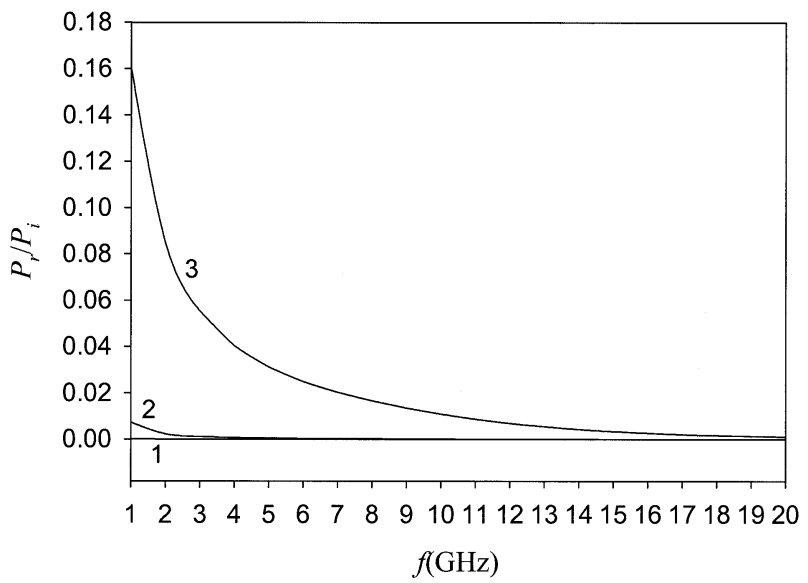

Fig. 4. Reflection ratio versus wave frequency, $\nu_{\mathrm{en}}=60 \mathrm{GHz}, B=2.5 \mathrm{kG}$ (1) $n_{c}=1 \times 10^{16} \mathrm{~m}^{-3}$. (2) $n_{c}=1 \times 10^{17} \mathrm{~m}^{-3}$. (3) $n_{c}=1 \times 10^{18} \mathrm{~m}^{-3}$.

The total absorbed power can be derived by subtracting the total reflecting power and transmitted power from the incident power

$$
P_{a}=P_{i}-P_{r}-P_{t}
$$

\section{RESULTS AND DISCUSSION}

In this section, we calculate and discuss the effects of the plasma parameters and background magnetic field on the reflected, transmitted, and absorbed power according to the previous equations. The ratio of the total reflected power to the incident power versus the electromagnetic wave frequency is first calculated. The electromagnetic wave frequency is taken from 1 to $20 \mathrm{GHz}$. The reflection ratio $P_{r} / P_{i}$ versus the wave frequency $f$ at different collision frequency $\nu_{\text {en }}$ is shown in Fig. 3. In this calculation, the plasma density in the center $n_{c}$ is $1 \times 10^{18} \mathrm{~m}^{-3}$ and the uniform magnetic field $B$ is $2.5 \mathrm{kG}$. The reflection ratios decrease with increasing collision frequency in the electromagnetic wave frequency regime of 1 to $20 \mathrm{GHz}$. The reflection ratio $P_{r} / P_{i}$ for different center plasma density $n_{c}$ at $\nu_{\mathrm{en}}=60 \mathrm{GHz}$ is plotted in Fig. 4. The reflection ratios increase with the center plasma density for electromagnetic wave frequency from 1 to $20 \mathrm{GHz}$. The reflection ratios are less

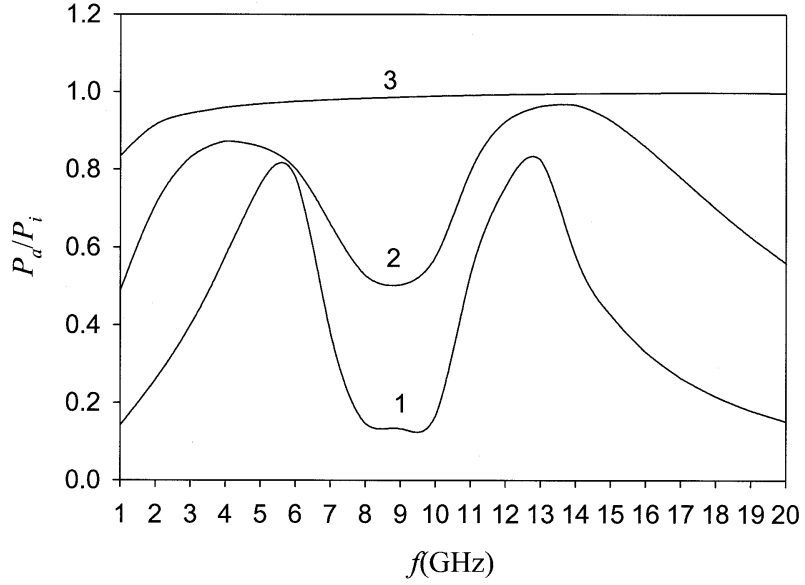

Fig. 5. Absorption ratio versus wave frequency, $n_{c}=1 \times 10^{18} \mathrm{~m}^{-3}, B=$ $2.5 \mathrm{kG}$. (1) $\nu_{\mathrm{en}}=1 \mathrm{GHz}$. (2) $\nu_{\mathrm{en}}=5 \mathrm{GHz}$. (3) $\nu_{\mathrm{en}}=60 \mathrm{GHz}$.

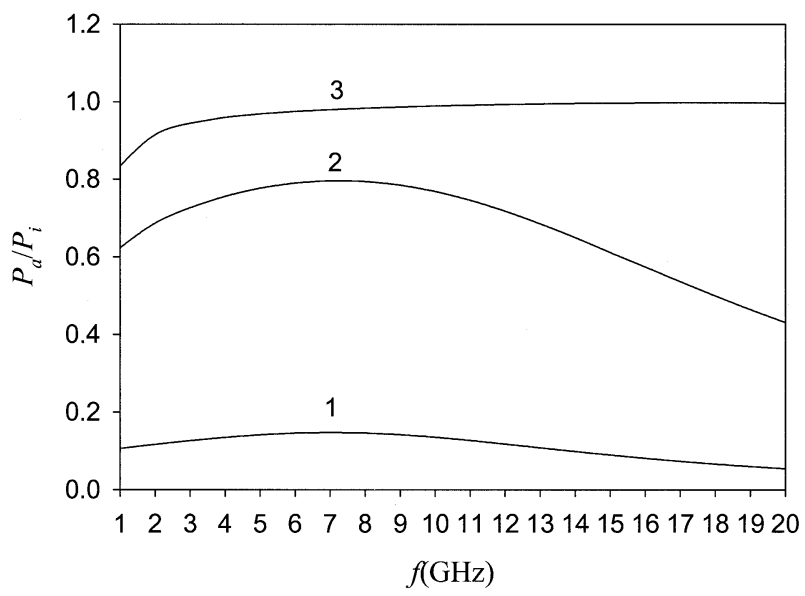

Fig. 6. Absorption ratio versus wave frequency, $\nu_{\mathrm{en}}=60 \mathrm{GHz}, B=2.5 \mathrm{kG}$. (1) $n_{c}=1 \times 10^{16} \mathrm{~m}^{-3}$. (2) $n_{c}=1 \times 10^{17} \mathrm{~m}^{-3}$. (3) $n_{c}=1 \times 10^{18} \mathrm{~m}^{-3}$.

than $0.7 \%$ at $n_{c}=1 \times 10^{17} \mathrm{~m}^{-3}$, as indicated by curve (2) in Fig. 4. It can be inferred that a low-density but highly collisional plasma can greatly reduce the amount of reflection power. The ratios of the total absorbed power to the incident power under different plasma conditions are then calculated. The absorption ratio $P_{a} / P_{i}$ versus the wave frequency at different plasma collision frequencies is displayed in Fig. 5. In this calculation, the center plasma density $n_{c}$ is $1 \times 10^{18} \mathrm{~m}^{-3}$ and the uniform magnetic field $B$ is $2.5 \mathrm{kG}$. The absorption ratios are more than $90 \%$ at $\nu_{\mathrm{en}}=60 \mathrm{GHz}$ for electromagnetic wave frequencies from 2 to $20 \mathrm{GHz}$. The absorption ratio $P_{a} / P_{i}$ versus the wave frequency at different center plasma density $n_{c}$ is exhibited in Fig. 6. Here, the plasma collision frequency $\nu_{\mathrm{en}}$ is $60 \mathrm{GHz}$. We see that the higher the plasma density, the larger is the absorption power as well as the broader the absorption bandwidth. It can, thus, be concluded that a higher number density and collision plasma will absorb the electromagnetic waves and increase the high-absorption bandwidth of electromagnetic waves. The above results demonstrate that magnetized nonuniform plasma may be used as broadband absorber of electromagnetic wave. However, the high density and collision frequency are difficult to achieve simultaneously. In addition, the reflection power is not expected to increase with plasma density. That is, the plasma 


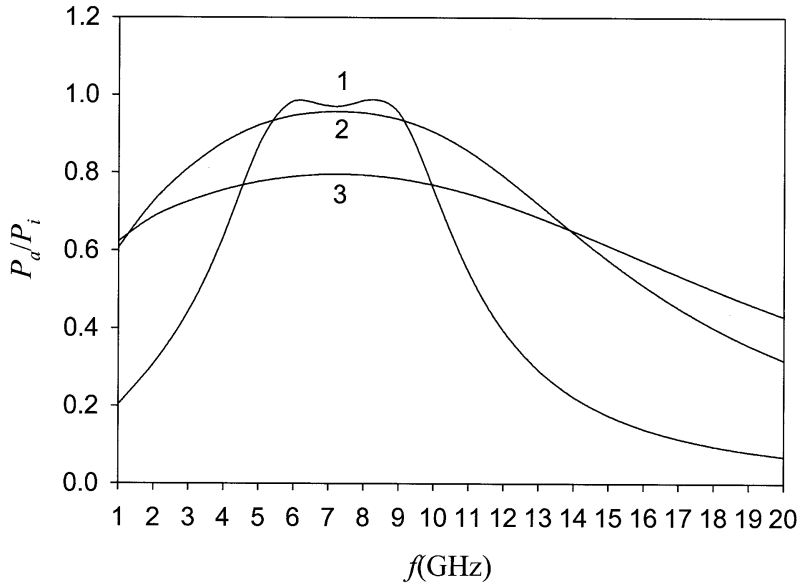

Fig. 7. Absorption ratio versus wave frequency, $n_{c}=1 \times 10^{17} \mathrm{~m}^{-3}, B=$ $2.5 \mathrm{kG}$. (1) $\nu_{\mathrm{en}}=5 \mathrm{GHz}$. (2) $\nu_{\mathrm{en}}=30 \mathrm{GHz}$. (3) $\nu_{c}=60 \mathrm{GHz}$.

parameters, such as plasma density and collision frequency, possess optimal values in electromagnetic wave absorption. Fig. 7 shows the absorption ratio at lower center plasma density for different collision frequencies. It is found that the absorption ratio $P_{a} / P_{i}$ decreases with increasing collision frequency in the range of $6-9 \mathrm{GHz}$ electromagnetic wave. This is an interesting result because it indicates that in some frequency bands, increasing the collision frequency does not necessarily benefit electromagnetic wave absorption. The low-density, low-collisional, and strong-magnetized plasma can also absorb greatly the electromagnetic wave power in the vicinity of the electron gyrofrequency. This can be explained by the following dispersion relation $\omega(k)$ of $\mathrm{R}$-wave [17] propagating in a collisional plasma:

$$
\frac{c^{2} k^{2}}{\omega^{2}}=1-\frac{\omega_{p}^{2}}{\omega\left[\left(\omega-\omega_{c e}\right)-j \nu_{\mathrm{en}}\right]} .
$$

When $\omega \rightarrow \omega_{c e}$, (15) can be expressed as

$$
\frac{c^{2} k^{2}}{\omega_{c e}^{2}}=1-\frac{\omega_{p}^{2}}{\omega_{c e} \nu_{\mathrm{en}}} j
$$

where $k$ is a complex wave number and can be written as $k=$ $\beta-j \alpha$. Therefore, (16) can be written as

$$
\beta^{2}-\alpha^{2}-2 \alpha \beta j=\frac{\omega_{c e}^{2}}{c^{2}}-\frac{\omega_{c e} \omega_{p}^{2}}{\nu_{\mathrm{en}} c^{2}} j .
$$

The attenuation coefficient $\alpha$ can be solved from (17)

$$
\alpha^{2}=\frac{-\frac{\omega_{c e}^{2}}{c^{2}}+\frac{\omega_{c e}^{2}}{c^{2}} \sqrt{1+\frac{\omega_{p}^{4}}{\omega_{c e}^{2} \nu_{\text {en }}^{2}}}}{2} .
$$

When $\omega_{p} \gg \nu_{\mathrm{en}}, \alpha \rightarrow \alpha_{0}$, where $\alpha_{0}=\left(\left(\omega_{c e} \omega_{p}^{2}\right) /\left(2 c^{2} \nu_{\mathrm{en}}\right)\right)^{1 / 2}$ is a large positive value ( $\alpha<0$ is nonphysical). As we know, (5) can also be expressed as

$$
\mathbf{E}=\mathbf{E}_{\mathbf{o}} e^{j(\omega t-k z)}=\mathbf{E}_{\mathbf{o}} e^{-\alpha z} e^{j(\omega t-\beta z)} .
$$

When $\omega \rightarrow \omega_{c e}$, the plane wave amplitude $\mathbf{E}_{\mathbf{0}} e^{-\alpha_{0} z}$ will be sharply attenuated with the increase of $z$ in the low-collisional

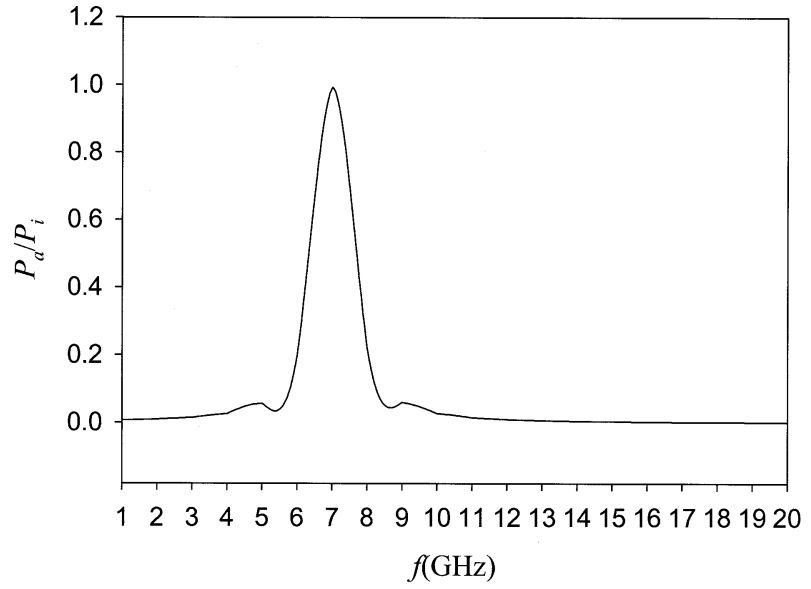

Fig. 8. Absorption ration versus wave frequency, $n_{c}=1 \times 10^{16} \mathrm{~m}^{-3}, \nu_{\mathrm{en}}=$ $1 \mathrm{GHz}$, and $B=2.5 \mathrm{kG}$.

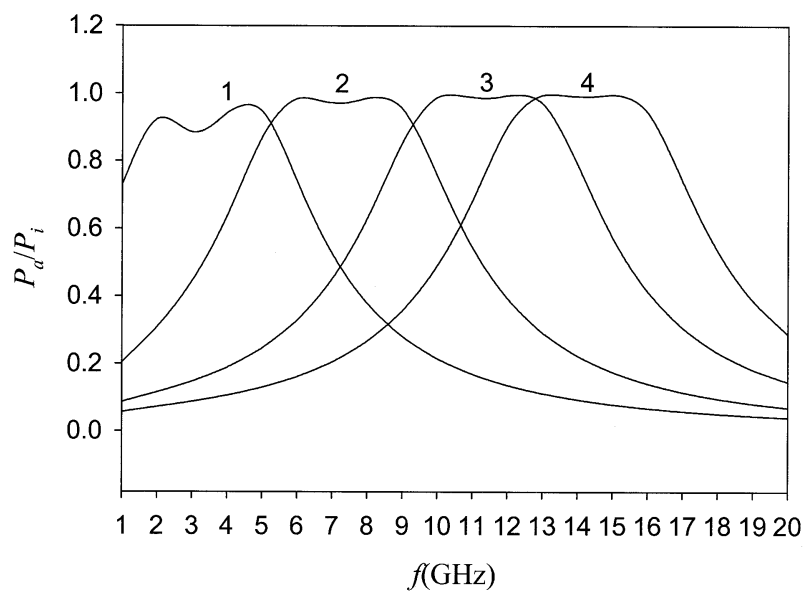

Fig. 9. Absorption ratio versus wave frequency, $n_{c}=1 \times 10^{17} \mathrm{~m}^{-3}, \nu_{\mathrm{en}}=$ 5 GHz. (1) $B=1$ kG. (2) $B=2.5 \mathrm{kG}$. (3) $B=4$ kG. (4) $B=5 \mathrm{kG}$.

plasma. This means that the high absorption of low-collisional magnetized plasma can be observed in the vicinity of electron gyrofrequency. The high-resonant absorption can be observed especially for low-density $\left(n_{c}=1 \times 10^{16} \mathrm{~m}^{-3}\right)$ and collisional $\left(\nu_{\mathrm{en}}=1 \mathrm{GHz}\right)$ magnetized plasma, as depicted in Fig. 8. Here, the magnetic field strength $B$ is $2.5 \mathrm{kG}$ and $\omega_{c e}=7 \mathrm{GHz}$. It is seen that the high-absorption peak occurs nicely in the electron gyrofrequency. From (18), it is also seen that the attenuation coefficient increases with the decrease of collision frequency. Therefore, the absorption ratio $P_{a} / P_{i}$ decreases with increasing collision frequency in the vicinity of the electron gyrofrequency, as shown in Fig. 7. In addition, the decrease of $P_{a} / P i$ with $\nu_{\mathrm{en}}$ is partly due to the increase of $P_{t}$.

In the calculation, the magnetic field strength is fixed. Fig. 9 shows the absorption ratio versus electromagnetic wave frequency for different magnetic field strengths. In the case of uniform magnetic field, $B$ is, respectively, $1,2.5,4$, and $5 \mathrm{kG}$. Here, the plasma density and collision frequency are $1 \times 10^{17} \mathrm{~m}^{-3}$ and $5 \mathrm{GHz}$, which can be easily achieved for atmospheric pressure plasma. Fig. 8 indicates that the resonant absorption band varies with the magnetic field strength. The resonant absorption band moves from low to high frequency as the magnetic field strength increases. Hence, the change of 


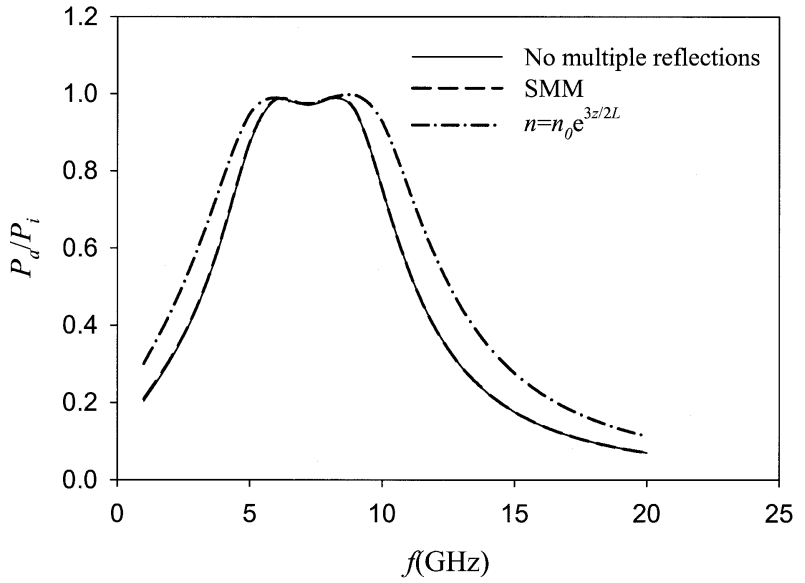

Fig. 10. Absorption ratio versus wave frequency, $\nu_{\mathrm{en}}=5 \mathrm{GHz}, B=2.5 \mathrm{kG}$. Solid line: No multiple reflections, $n_{c}=1 \times 10^{17} \mathrm{~m}^{-3}$. Dashed line: SMM, $n_{c}=1 \times 10^{17} \mathrm{~m}^{-3}$. Dashed-dotted line: $n=n_{0} e^{3 z / 2 L}, n_{0}=5 \times 10^{16} \mathrm{~m}^{-3}$.

magnetic field strength will obviously affect the high-resonant absorption frequency. One reason is that the gyrofrequency increases with the magnetic field strength. It, thus. indicates that low-density and low-collision magnetized nonuniform plasma can also be used as broadband absorption of electromagnetic waves through the change of the magnetic field strength.

For comparison, the plasma slab is calculated according to the SMM full analysis of nonuniform magnetized plasma slab [13] which is also divided into 12 adjacent subslabs with the same electron density gradient in order to obtain accurate calculation results. The calculation results are depicted in Fig. 10. It is seen that the absorption ratio $P_{a} / P_{i}$ of the plasma with no multiple reflections is in good agreement with the one with SMM analysis. The exponential profile $\left(n=n_{0} e^{3 z / 2 L}, n_{0}=\right.$ $5 \times 10^{16} \mathrm{~m}^{-3}$ ) of the electron density has also been studied to investigate the influence of the density gradient on the absorption characteristics of magnetized plasma, as shown by the dash line in Fig. 10. The high-absorption band is broader than the parabolic profile with the same center electron density, implying that the electron density gradient will also affect the plasma absorption characteristics especially the broadband absorption property.

\section{CONCLUSION}

The absorption, reflection, and transmission of electromagnetic waves by nonuniform plasma confined in an external uniform magnetic field are calculated under different plasma conditions and magnetic field strengths. The electromagnetic wave frequency is from 1 to $20 \mathrm{GHz}$. A low-density but high-collision plasma can greatly reduce the reflection power, whereas high plasma density and collision plasma can increase the bandwidth of absorption of electromagnetic wave. The effects of magnetic field strength and density gradient on the absorbed power as well as the frequency band of resonant absorption are very significant. More than $90 \%$ of the electromagnetic wave power can be absorbed in high-density and high-collision plasma. The broadband absorption of electromagnetic waves can also be achieved in low-density and collision plasma in the presence of an external uniform magnetic field of various strengths. The calculation results are corroborated by the SMM analysis of absorption characteristics of nonuniform magnetized plasma slab. The results show that magnetized nonuniform plasma has better properties with regard to broadband absorption of electromagnetic waves.

\section{REFERENCES}

[1] X. M. Qiu, "Interaction of electromagnetic wave with plasma" (in Chinese), Nucl. Fusion Plasma Phys., vol. 18, pp. 53-55, 1998.

[2] V. L. Ginzberg, The Propagation of Electromagnetic Waves in Plasma. New York: Pergammon, 1970.

[3] N. S. Yoon, D. I. Choi, and Hanbit Project Team, "Interaction of a transverse electromagnetic wave with a bounded thermal plasma with an external magnetic field," in Proc. 25th EPS Conf. Control Fusion and Plasma Physics, Prague, Czech Republic, 1998, p. 364.

[4] R. J. Vidmar, "On the use of atmospheric plasmas as electromagnetic reflectors and absorbers," IEEE Trans. Plasma Sci., vol. 18, pp. 733-741, Aug. 1990.

[5] J. Santoru, D. J. Gregoire, and R. W. Schumacher, "Electromagnetic wave absorption in unmagnetized plasmas," Bull. Amer. Phys. Soc., vol. 35, p. 2067, 1990.

[6] W. W. Destle, J. E. Degrange, H. H. Fleischmann, J. Rodgers, and Z. Segalov, "Experimental studies of high power microwave reflection, transmission, and absorption from a plasma-covered plane boundary," J. Appl. Phys., vol. 69, pp. 6313-6318, 1991.

[7] M. Laroussi and J. Reece Roth, "Numerical calculation of the refletion, absorption and transmission of microwaves by a nonuniform plasma slab," IEEE Trans. Plasma Sci., vol. 21, pp. 366-372, Aug. 1993.

[8] M. Laroussi, "Interaction of microwave with atmospheric pressure plasmas," Int. J. Infrared Millim. Waves, vol. 16, pp. 2069-2083, 1995.

[9] E. Koretzky and S. P. Kuo, "Characterization of an atmospheric pressure plasma generated by a plasma torch array," Phys. Plasmas, vol. 5, pp. 3774-3780, 1998.

[10] H. M. Liu, X. W. Hu, Z. H. Jiang, X. P. Lu, C. L. Gu, and Y. Pan, "Electromagnetic wave attenuation in atmospheric pressure plasmas," Chin. Phys. Lett., vol. 18, pp. 1225-1226, 2001.

[11] M. A. Heald and C. B. Wharton, Plasma Diagnostics With Microwaves. New York: Krieger, 1978, pp. 71-94.

[12] G. T. Ruch, D. E. Barrick, and C. K. Krichbaum, Radar Cross Section Handbook. New York: Plenum, 1970, pp. 473-492.

[13] J. A. Kong, Electromagnetic Wave Theory. New York: Wiley, 1986, pp. $110-132$.

[14] B. J. Hu, G. Wei, and S. L. Lai, "SMM analysis of reflection, absorption and transmission from nonuniform magnetized plasma slab," IEEE Trans. Plasma Sci., vol. 27, pp. 1131-1136, Aug. 1999.

[15] J. R. Roth, "Microwave absorption system," United States Patent, no. 4989006 , Jan. 29, 1991.

[16] M. Laroussi, "A tunable microwave notch absorber filter," Int. J. Infrared Millim. Waves, vol. 13, pp. 1557-1569, 1992.

[17] R. J. Goldston and P. H. Rutherford, Introduction to Plasma Physics. London, U.K.: IOP, 1995.

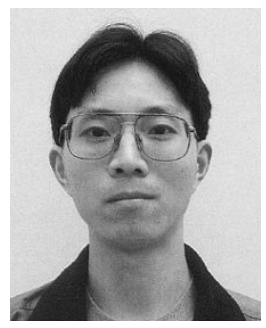

D. L. Tang was born in Sichuan, China, 1969. He received the B.S. degree from Xi'an Jiaotong University, Xi' an, China, and the M.S. degree from the Southwestern Institute of Physics, Chengdu, China, where he is currently working toward the Ph.D. degree.

He is currently an Associate Research Professor with the Southwestern Institute of Physics and a Research Associate in the Plasma Laboratory, City University of Hong Kong, Kowloon. His research interests include plasma physics and technology, electromagnetic propagation, and microwave technique. 


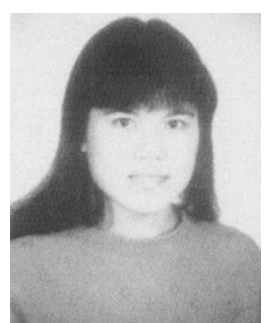

A. P. Sun was born in Hunan, China, in 1969. She received the B.S. degree from the Xiangtan Normal College, Hunan, China, the M.S. degree from the Sichuan University, Sichuan, China, and the Ph.D. degree from the Southwestern Institute of Physics, Chengdu, China.

She is currently an Associate Research Professor with the Southwestern Institute of Physics. Her research interests include plasma theory, electromagnetic propagation, and microwave technique.

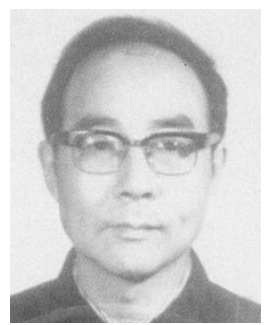

X. M. Qiu was born in Hubei, China, 1938. He graduated from Peking University, Beijing, China, in 1959.

Since August 1987, he has been a Research Professor with Southwestern Institute of Physics, Chengdu, China. He has been a Visiting Professor with the Department of Modern Physics, University of Science and Technology, Hefei, China, since 1985. His research interests include plasma theory, chaos and soliton theory, and high- $T c$ superconductivity theory.

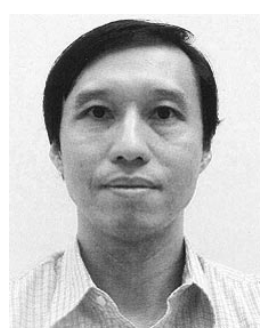

Paul K. Chu (M'97-SM'99-F'03) received the B.S. degree in mathematics from The Ohio State University, Columbus, in 1977, and the M.S. and Ph.D. degrees in chemistry from Cornell University, Ithaca, NY, in 1979 and 1982, respectively.

He joined Charles Evans and Associates, Redwood City, CA, in 1982, and started his own business in 1990. He is currently Professor (Chair) of materials engineering with the Department of Physics and Materials Science, City University of Hong Kong, Hong Kong. He also holds concurrent Professorships at Fudan University, Shanghai, Peking University, Peking, China, Southwest Jiaotong University, Chengdu, and the Southwestern Institute of Physics, Chengdu, China. His research activities include plasma-processing technology, microelectronics processing, and materials characterization. He is author/coauthor of 450 publications, has seven U.S. patents, and serves on the Editorial Board of Nuclear Instruments and Methods in Physics Research B.

Dr. Chu is a Fellow of the Hong Kong Institute of Engineers (HKIE) and an executive member of the International Plasma-Based Ion Implantation Committee. He also serves on the Engineering Panel of the Hong Kong Research Grants Council (RGC). 